\title{
CONTRIBUTION A L'ÉTUDE DU DOSAGE DE LA LYSINE " DISPONIBLE " DES LAITS ECRÉMÉS EN POUDRE AU MOYEN DU FLUORODINITROBENZENE
}

\author{
R. PION \\ Service de Biochimie et de Nutrition, \\ Centre national de Recherches zootechniques, Jouy-en-Josas.
}

\section{SOMMAIRE}

L'application aux laits écrémés en poudre du dosage de la lysine "disponible " au moyen du fluorodinitrobenzène a été étudiée. Deux méthodes ont été comparées : celle de Carpenter et Jí. LINGer (I955) et celle de Schober et PRINZ (I956).

Une modification de cette dernière méthode a été finalement utilisée pour comparer les teneurs en lysine " disponible " ainsi déterminées aux teneurs en lysine totale obtenues par dosage microbio. logique après hydrolyse acide.

Il n'y: avait de différence significative entre teneur en lysine " disponible " et teneur en lysine totale que pour une poudre de lait très altérée, colorée et peu soluble.

\section{IN'TRODUCTION}

De nombreux auteurs ont montré que les protéines de laits peuvent être altérées, soit au cours de la fabrication des laits en poudre, soit pendant leur conservation, et que cette altération pouvait se traduire en particulier par une diminution de la quantité de lysine libérable par les enzymes digestifs, due à un blocage du groupement $\varepsilon$-aminé normalement libre dans la protéine.

L'influence du procédé de fabrication a été étudiée en particulier par MAURON et al. (I955) par dosage de la lysine libérée par les enzymes protéolytiques et de la lysine totale ; tandis que la conservation a été notamment l'objet d'une longue étude de HenRY et al. (I948), où les résultats de nombreux dosages chimiques et de tests biologiques sont comparés.

Aussi était-il intéressant d'étudier les conditions dans lesquelles la méthode de dosage de la lysine " disponible ", au moyen du fluoro-2. 4-dinitrobenzène, préconisée par CARPENTER et EILINGER (I955), en particulier pour différentes farines animales, et étendue par BRUNo et CARPENTER (I957) à l'examen des aliments protéiques végétaux, était applicable aux laits en poudre.

Cette méthode utilise la réaction du fluorodinitrobenzène (FDNB) sur les groupements aminés libres des protéines. La dinitrophénylprotéine obtenue (DNP protéine) est hydrolysée, et la majorité des dinitrophénylaminoacides (DNP aminoacides) 
sont extraits à l'éther, ainsi que l'excès de FDNB éventuellement présent. L' $\varepsilon$ dinitrophényl-1ysine ( $\varepsilon$ DNP 1ysine) reste dans la phase aqueuse et est dosée photométriquement. On obtient ainsi un dosage de la lysine dont le groupement $\varepsilon$ aminé est resté libre.

Une méthode similaire a été appliquée aux laits en poudre par ScHOBER et PrINz (I956). Malheureusement, les conditions d'hydrolyse préconisées par ces auteurs sont difficilement utilisables pour des analyses de série.

Aussi avons-nous comparé les conditions opératoires proposées par CARPENTER et ELLINGER à celles que préconisent SCHOBER et PRINZ à l'exception de la méthode d'hydrolyse, et les résultats ainsi obtenus aux teneurs en lysine totale.

\section{MATÉRIEL E'T MÉTHODES}

\section{A. - ÉCHANTILLONS}

Les laits en poudre utilisés pour les essais étaient soit des laits en poudre séchés par pulvérisation (laits Spray), soit des laits séchés sur cylindres chauffés à la vapeur (laits Hatmaker).

Lait $n^{0}$ I poudre Spray

- pasteurisée à $87^{\circ} \mathrm{C}$,

- concentrée à $45 \mathrm{p}$. roo de matière sèche dans un appareil WIEGAND à $54^{\circ} \mathrm{C}$,

- séchée dans une tour Niro Atomizer à $92^{\circ} \mathrm{C}$,

- conservée à $+4^{\circ} \mathrm{C}$.

- Laits $n^{0} 2$ et $n^{0} 3$ poudres Spray:

- conditions de fabrication inconnues,

- conservées à $+4^{\circ} \mathrm{C}$

- Lait $n^{0} 4$ poudre Hatmaker :

- pasteurisée à $75^{\circ} \mathrm{C}$

- séchée sur cylindres chauffés à la vapeur (pression $6 \mathrm{~kg} / \mathrm{cm}^{2}$ ),

- conservées à $+4^{\circ} \mathrm{C}$.

- Laits $n^{\mathrm{os}} 5$ - 6 et 7 poudres Hatmaker :

- conditions de fabrication inconnues,

- conservées pendant 6 mois à l'usine dans les conditions habituelles utilisées par le fabricant, puis à $+4^{\circ} \mathrm{C}$.

Tous ces échantillons sont des laits écrémés.

\section{B. - RÉACTIFS}

— solution de bicarbonate de sodium à ıo p. roo (p/p),

- éthanol $95^{\circ}$ redistillé ;

- méthanol redistillé ;

- solution de FDNB à 2,5 p. $100(\mathrm{v} / \mathrm{v})$ dans l'éthanol;

- solution de FDNB à Io p. roo (v/v) dans le méthanol (ces deux solutions sont préparées immédiatement avant usage) (1) ;

- acide chlorhydrique $6 \mathrm{~N}$;

- éther éthylique ;

- lessive de soude à $40 \mathrm{p}$. $100(\mathrm{p} / \mathrm{p})$;

- acide chlorhydrique $\mathrm{N}$;

- soude $\mathrm{N}$;

- solution de bicarbonate de sodium à I p. Ioo (p/p) ;

- solution de bicarbonate de sodium à 2 p. $100(\mathrm{p} / \mathrm{p})$;

- acide acétique glacial;

- acétone ;

(1) Il est dangereux de pipetter ces solutions. 
- solvant pour chromatographie : alcool amylique tertıaire saturé de tampon phtalate $(50 \mathrm{ml}$ de phtalate acide de potassium o, I $\mathrm{M}+45,5 \mathrm{ml}$ de soude o, $\mathrm{N}$ amenés à roo $\mathrm{ml}$ ).

Le FDNB provenait de L Light and Co. Les autres produite, sauf les alcools, étaient des produits R. P. Prolabo.

\section{C. -- MÉTHODES}

$\mathrm{I}^{\circ}$ Réaction du lil)NB sur la poudre de lait.

Deux méthodes ne différant que par le réactif, ont été utilisées.

- Méthode I (Carpenter et Eulinger, i955) (Carpenter et al., i957).

La prise d'essai ( $100 \mathrm{mg}$ ), placée dans un tube de centrifugeuse muni d'un rôdage normalisé, est mise en suspension dans une solution de bicarbonate de sodium à ro p. $100(2,5 \mathrm{ml})$, puis laissée au repos pendant to minutes. On ajoute alors $4 \mathrm{ml}$ de la solution à $2,5 \mathrm{p}$. I00 de FDNB dans l'éthanol (réactif I). tif.

Jes tubes sont bouchés et agités longitudinalement au moyen d'un agitateur mécanique alterna-

Sauf indication contraire, le temps d'agitation a été de deux heures.

- Méthodo 11 (SCHOBER et P'RINZ, I956).

Le mode opératoirc est le même que ci-dessus, mais on utilise $3 \mathrm{ml}$ de solution de FDNB à Io p. 100 diuns le méthanol. Les blancs sont traités de la même manière, en l'absence de FDNB.

20 Iriparation à lhydrolyse.

-. Mithode $I$.

J.éthanol ext évalporé au bain-marie jusqu'ì ce que l'ébullition cesse.

-.. Mithode II.

La INP protéine est séparée du méthanol et de l'excès de FDNB par centrifugation. Pour cela, on rend la teneur en méthanol du mélange supéricure à $9 \circ \mathrm{p}$. I 100 , et l'on centrifuge à 1700 g environ pendant ıo minutes. Lescumageant est éliminé, et le précipité lavé avec to ml de méthanol. Le méthanol ess à noureau élininé par centrifugation. Cette opération est répétée 4 fois. Sauf indication contraire, les centrifugations ont été effectuées dans une centrifugeuse refroidie à $4^{\circ} \mathrm{C}$ environ.

\section{$3^{\circ}$ Hydrolyse de la DNP proléine.}

'Toutes les hydrolyses ont été effectuées à reflux, au bain d'huile à $120^{\circ} \mathrm{C}$, dans l'acide chlorhydrique $6 \mathrm{~N}$ ( $8 \mathrm{ml}$ pour $100 \mathrm{mg}$ de lait en poudre). Pour cela, des réfrigérants à air munis de rôdages sont adaptés aux tubes de centrifugeuse.

Nous n'avons pas utilisé le mélange préconisé par SchObER et Prinz (acide formique, anhydride acétique, acide chlorhydrique concentré $90-5,5-60$ ) qui nécessite l'usage d'une bombe.

$4^{\circ}$ Séparation de l'e DNP lysine.

Après filtration des hydrolysats, une fraction aliquote (le dixième du total) est extraite par 4 volumes d'éther éthylique. Pour les échantillons traités selon la méthode I, 6 extractions ont été nécessaires pour que le test indiqué par CARPENTER et al. (coloration jaune obtenue en agitant l'extrait éthéré avec NaOH 40 p. Ioo) soit négatif. Dans le cas de la méthode II, où les hydrolysats sont en principe exempts de FDNB, 3 extractions se sont révélées suffisantes, d'après les critères chromatographiques utilisés. La phase aqueuse et les lavages de l'ampoule à décanter (un lavage à l'acide chlorhydrique $N$, suivi de 3 lavages à l'eau) sont transférés dans des béchers ; l'éther dissous est éliminé par un passage de quelques minutes au bain de vapeur.

\section{$5^{\circ}$ Dosage photomítrique}

\section{a) Préparation de l'є DNP lysine.}

L'є DNP lysine, destinée à la confection des solutions standard, a été préparée selon PoRTER et SANGER (I948). Conservé à l'obscurité, ce composé s'est révélé stable pendant au moins un an.

\section{b) Solutions standard.}

L' $\varepsilon$ DNP lysine a été dissoute soit dans le bicarbonate de sodium I p. roo, soit dans une solution tamponnée à pH 5 par l'acide acétique glacial et la soude $40 \mathrm{p}$. IOO (CARPENTER et ELLINGER, 1955).

\section{c) Dosage de l'є DNP lysine des hydrolysats.}

A la suite de l'étude des solutions étalons, c'est l'utilisation du bicarbonate de sodium I p. Ioo qui a été choisie pour effectuer les lectures. Après élimination de l'éther dissous, les extraits aqueux 
sont neutralisés par la soude $\mathrm{N}$. Ils sont alors mélangés volume à volume avec la solution de bicarbonate de sodium à $2 \mathrm{p}$. 100. Les densités optiques sont mesurées au spectrophotomètre JobIN et Yvon.

$6^{\circ}$ Chromatographie qualitaitve des solutions obtenues après exiraction à l'éther (Levy, 1955).

Après extraction à l'éther, les hydrolysats sont évaporés à sec et repris par l'acétone. L' $\varepsilon$ DNP lysine est séparée des autres constituants colorés éventuellement présents par une migration de 24 heures en chromatographie descendante au moyen du solvant alcool anylique tertiaire saturé de tampon phtalate de $\mathrm{Na}$ pH 6, sur papier Whatman 4 lavé avec ce tampon.

$7^{\circ}$ Dosage de la lysine totale.

La lysine totale est dosée par voie microbiologique après hydrolyse par l'acide chlorhydrique $6 \mathrm{~N}$ à $\cos ^{\circ} \mathrm{C}$ pendant I 2 heures. Nous avons vérifié que les résultats oltenus étaient identiques quand le temps d'hydrolyse variait de 8 heures it 15 heures.

On utilise Leuconostoc mesenteroides P 60 ATC 8042 et le milieu employé pour le dosage de la cystine par Cinevillard et al. (1952). Les hydrolysats sont filtrés à pII 3,0 (Fauconniale, I954). On opère sur $3 \mathrm{ml}$ de milieu final et le temps d'incubation est d'environ 72 heures (Dunv et al. 1947). L'acidité développée est mesurée par titrimétrie.

\section{RÉSULTATS E'T DISCUSSION}

\section{A. - DOSAGES PHOTOMÉTRIQUES}

L, spectre de l'e DNP lysine dans le bicarbonate I p. Ioo (figure I) présente un maximum d'absorption à $362 \mathrm{~m} \mu$. Les valeurs obtenues en milieu tamponné

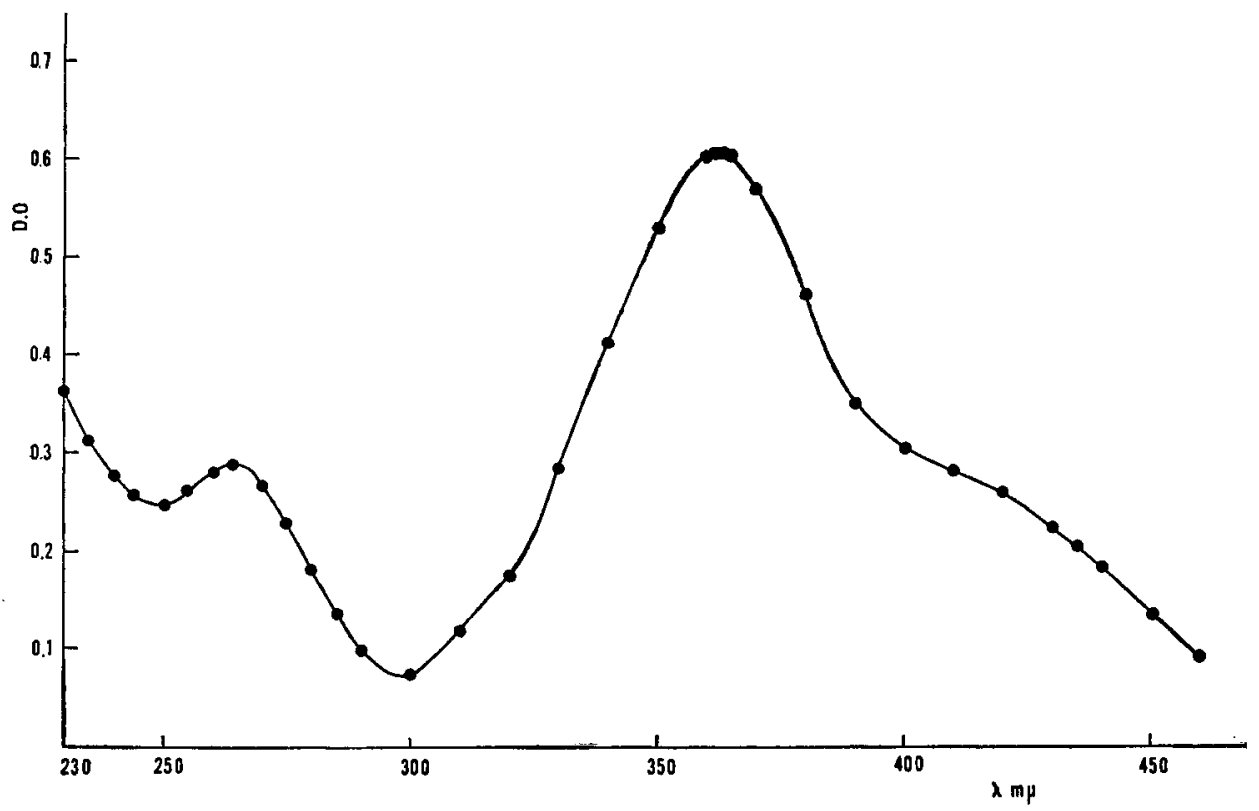

FIG. I -- Spectre d'absorpiton de l'ミDNP lysine (solution à 33 micromoles par litre dans le bicarbonate de Na 1 p. 100).

à $\mathrm{pH} 5$ par l'acétate de sodium sont très proches de celles obtenues en bicarbonate de sodium I p. Ioo, mais sont sensibles à la quantité d'acide acétique utilisé. Par contre, les coefficients d'extinction obtenus en milieu bicarbonate varient peu avec la con- 
centration : les résultats sont les mêmes que la solution de bicarbonate soit à $\mathbf{I}$ p. Ioo ou à $I, 5$ p. Ioo. Une quantité de chlorure de sodium correspondant à celle qui provient dans les échantillons de la neutralisation de l'acide chlorhydrique n'influe pas sur les densités optiques.

Courbes standard. - Les courbes standard (figure 2) ont été établies pour des concentrations variant de $I 8$ à 60 micromoles par litre de bicarbonate de sodium à I p. Ioo.

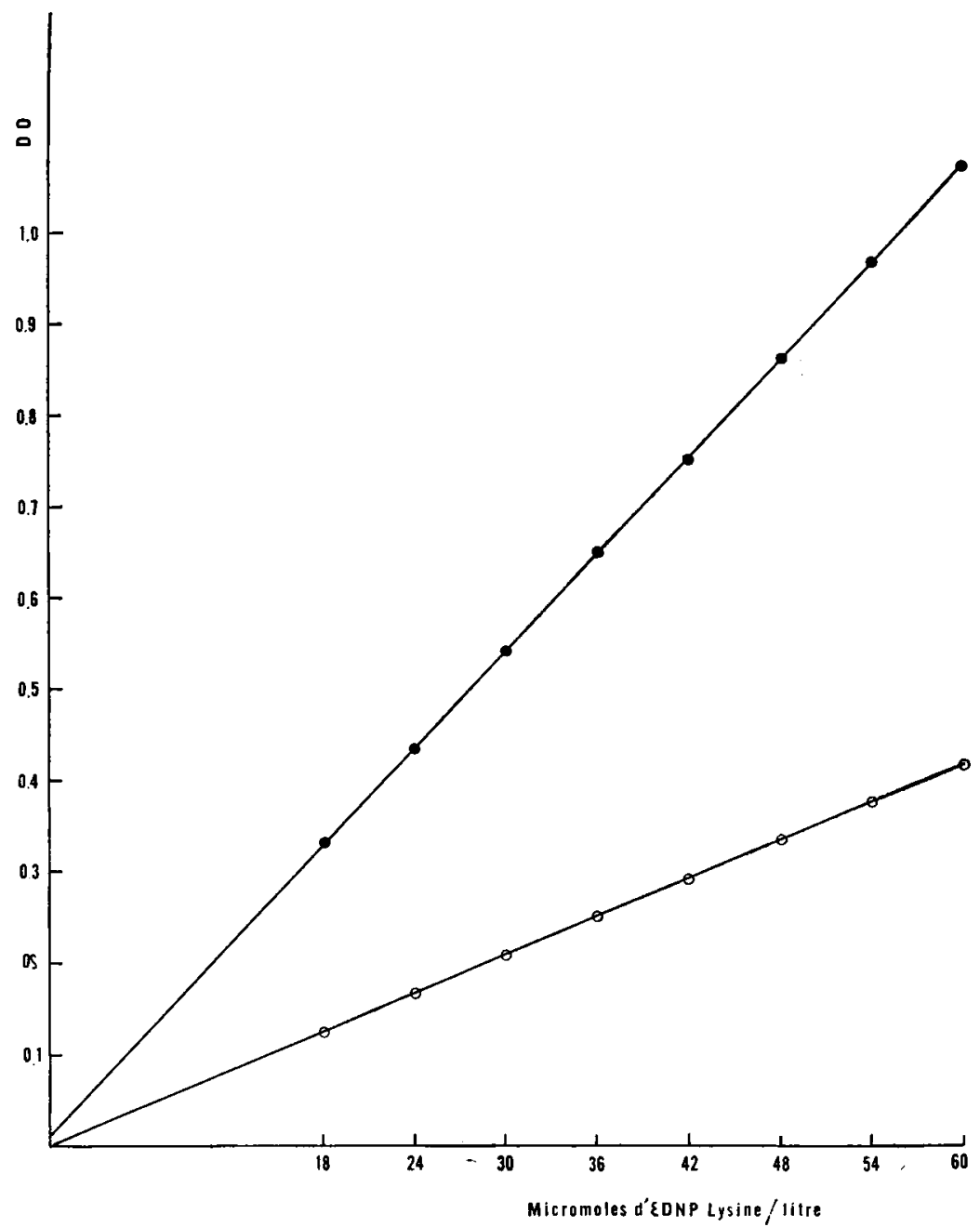

FIG. 2 - Courbes d'absorption de l'sDNP lysine en fonction de la concentration (dans $e$ bicarbonate de $N a$ à 1 p 100)

$\lambda=362 \mathrm{~m} \mu \quad O \lambda=435 \mathrm{~m} \mu$

A $362 \mathrm{~m} \mu$, la coloration suit la loi de Beer jusqu'à des densités optiques de l'ordre de $\mathbf{I}$.

A $435 \mathrm{~m} \mu$, la courbe standard est rectiligne dans la même zone de concentra- 
tion, mais la sensibilité est beaucoup plus faible, et une précision de lecture équivalente nécessite une concentration minimum de l'ordre de 40 micromoles par litre.

La plupart des mesures ont été effectuées à la fois à 362 et à $435 \mathrm{~m} \mu$, ce qui peut donner une meilleure appréciation de la pureté des solutions dosées.

\section{B. - RÉSUlTATS OBTLNUS PAR LES MÉTHODES I ET II, POUR UN MỀMÉ ÉCHANTILLON}

Les teneurs en lysine "disponible " obtenues pour le lait $n^{0}$ I par les méthodes I et II figurent dans le tableau $r$, et les valeurs moyennes des blancs obtenues dans les mêmes conditions sans FDNB dans le tableau 2. Ces valeurs ont été calculées à partir des lectures faites aux 2 longueurs d'ondes utilisées.

\section{TABLEAU I}

Comparaison des résultats obtenus par les méthodes $I$ et II

Lait $\mathrm{n}^{\circ} 1$ (Lysine totale 3,2 p. 100). Agritation : 2 heures

\begin{tabular}{|c|c|c|c|c|}
\hline \multirow{3}{*}{$\begin{array}{c}\text { Temps } \\
\text { d'hydrolyse }\end{array}$} & \multicolumn{4}{|c|}{ Lysine " disponible" p. 100} \\
\hline & \multicolumn{2}{|c|}{ Méthode 1} & \multicolumn{2}{|c|}{ Méthode II } \\
\hline & Lecture : $362 \mathrm{~m} \mu$ & Lecture : $4335 \mathrm{~m} \mu$ & Lecture : $362 \mathrm{~m} \mu$ & Lecture $: 435 \mathrm{~m} \mu$ \\
\hline \multirow{2}{*}{$14 \mathrm{~h}$. } & 2,62 & 2,82 & 3,09 & 2,75 \\
\hline & $2,8 j$ & 3,09 & 3,08 & 2,74 \\
\hline \multirow[t]{2}{*}{$16 \mathrm{~h}$. } & 2,95 & 2,97 & 2,89 & 2,57 \\
\hline & 3,04 & 3,17 & 3,06 & 2,77 \\
\hline \multirow[t]{2}{*}{$20 \mathrm{~h}}$. & 3,01 & 3,21 & 3,15 & 2,86 \\
\hline & 2,95 & 3,18 & 3,15 & 2,80 \\
\hline \multirow[t]{2}{*}{$24 \mathrm{~h}$. } & 2,29 & & 2,93 & \\
\hline & 2,46 & & 3,06 & \\
\hline
\end{tabular}

TABLEAU 2

Valeur moyenne des "Blancs"

\begin{tabular}{|c|c|c|c|c|}
\hline & \multicolumn{2}{|c|}{ Méthode I } & \multicolumn{2}{|c|}{ Méthode II } \\
\hline & Lecture : $362 \mathrm{~m} \mu$ & Lecture : $435 \mathrm{~m} \mu$ & Lecture : $362 \mathrm{~m} \mu$ & Lecture : $435 \mathrm{~m} \mu$ \\
\hline $\begin{array}{l}\text { Blancs : g lysine } \mathrm{p} . \\
\text { Ioo g lait ..... } \\
\text { Blancs p. Ioo de Ia } \\
\text { lysine dosée .... }\end{array}$ & $\begin{array}{l}0,44 \\
16\end{array}$ & $\begin{array}{l}0,6 \mathrm{I} \\
20\end{array}$ & $\begin{array}{l}0,11 \\
4\end{array}$ & $\begin{array}{l}0,16 \\
6\end{array}$ \\
\hline
\end{tabular}

Les résultats obtenus par la méthode II à $362 \mathrm{~m} \mu$ ne semblent pas dépendre du temps d'hydrolyse entre 14 heures et 24 heures.

Pour la méthode I, au contraire, les résultats sont un peu faibles après I4 heures d'hydrolyse, très faibles après 24 heures, les valeurs obtenues pour I6 heures et 20 heures étant comparables. 
Les valeurs des blancs sont beaucoup plus élevées pour la méthode I que pour la méthode II.

\section{C. - PURETÉ DES SOluTiONS DOSÉES}

Spectre d'absorption. - I es spectres des solutions obtenues pour le dosage par les méthodes I et II figurent respectivement sur les figures 3 et 4 .

Pour la méthode I, le spectre est nettement déformé par rapport à celui de la solution étalon, tandis que l'accord est assez bon pour la méthode II.

Les résultats obtenus à $435 \mathrm{~m} \mu$ sont toujours supérieurs à ceux obtenus à 362 mu pour la méthode $I$ ( $+6 \mathrm{p}$. Ioo en moyenne) tandis que le phénomène est inversé pour la méthode II (- Io p. IOo).

Toutefois, les densités optiques obtenues à 435 mu étaient trop faibles (de l'ordre de $0, I_{50}$ ), et les blanes trop élevés en valeur relative pour permettre une grande précision. De plus, la longueur d'onde de $435 \mathrm{~m} \mu$ ne correspond pas à un point singulier du spectre.

Chromatographie. - Les résultats sont les suivants :

Méthode I. - Après 24 heures de migration, on constate la présence de deux taches jaunes, l'une migrant comme l' $\varepsilon$ I)NP lysine étalon, l'autre ayant un Rf 2,7 fois plus élevé.

Méthode II. - Il n'y a qu'une seule tache colorée, ne se séparant pas de l'є DivP lysine étalon posée en surcharge.

I'ensemble de ces résultats montre que, si les valeurs obtenues par les méthodes I et II pour des temps d'hydrolyse de I 6 heures à 20 heures sont assez comparables la quantité d' $\varepsilon$ DNP lysine réellement présente dans les solutions obtenues par la méthode I est en fait plus faible, puisque ces solutions sont un mélange en proportions inconnues d' $\varepsilon$ IINP lysine et d'un autre composé.

\section{D. - ISSAI DU RÉAC’́TIF I, DANS LES CONDITIONS DE LA MÉTHODE II}

Dans la méthode I, l'hydrolyse s'effectue en présence d'un excès de FJONB, ce qui explique la spécifité insuffisante des extractions à l'éther effectuées. D'autre part l'élimination de l'éthanol est mal contrôlée.

Étant donné la faible quantité d'azote non protéique contenue dans le lait, le ou les composés interférents sont vraisemblablement produits en majeure partie au cours de l'hydrolyse (la deuxième tache trouvée sur les chromatogrammes a un $\mathrm{Rf}$ trop élevé pour être de la DNP arginine). Aussi, pour tester l'emploi du réactif I, l'utilisation des conditions opératoires de la méthode II (méthode III) a-t-elle été préférée à l'amélioration de la spécificité de l'extraction à l'éther proposée par Bruno et CARPENTER (I957), plus intéressante dans le cas d'un produit riche en acides aminés libres.

Les résultats obtenus pour un temps d'agitation de 2 heures figurent au tableau III. On constate une différence importante entre les séries I et II pour lesquelles tous les facteurs contrôlés étaient les mêmes. Cette variabilité est d'autant plus notable que, dans le cas de la méthode II, les valeurs obtenues après 24 heures d'hydrolyse sont très voisines de celles obtenues pour d'autres temps d'hydrolyse, alors qu'elles proviennent d'une autre série de dosages. 


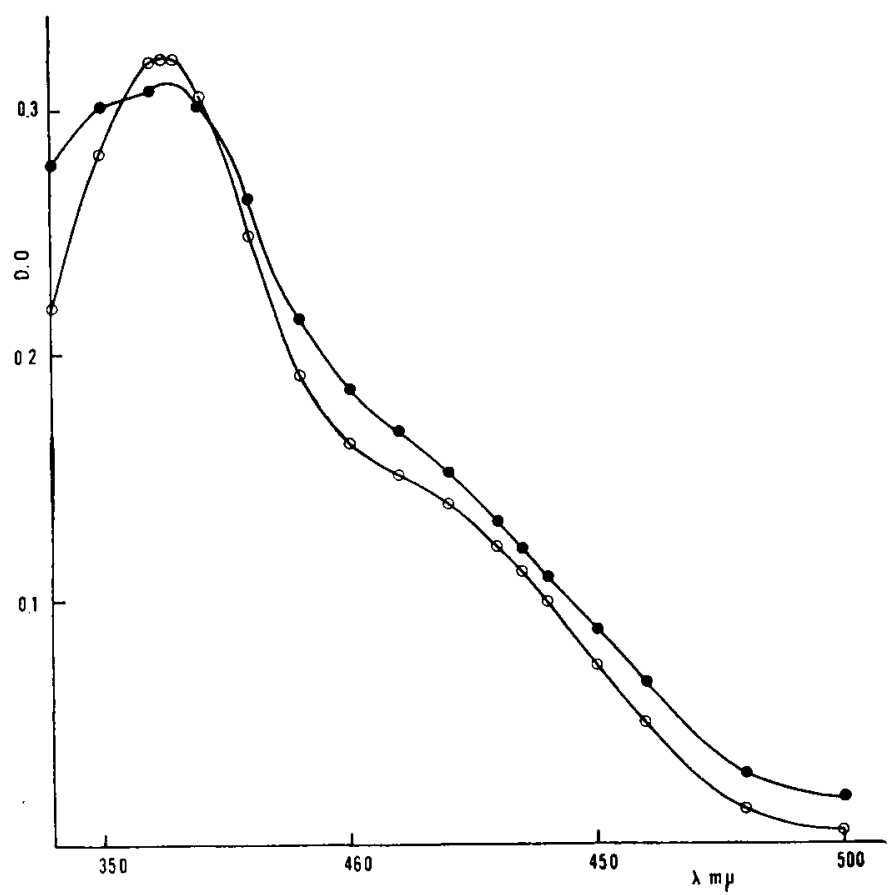

FIG. 3 - Spectres d'absorption de l'sDNP lysine et de la solution obtenue pour le dosage par la métho:le l (échantillon $n^{\circ} 4$ )

. EDNP lysine $\quad$. Solution

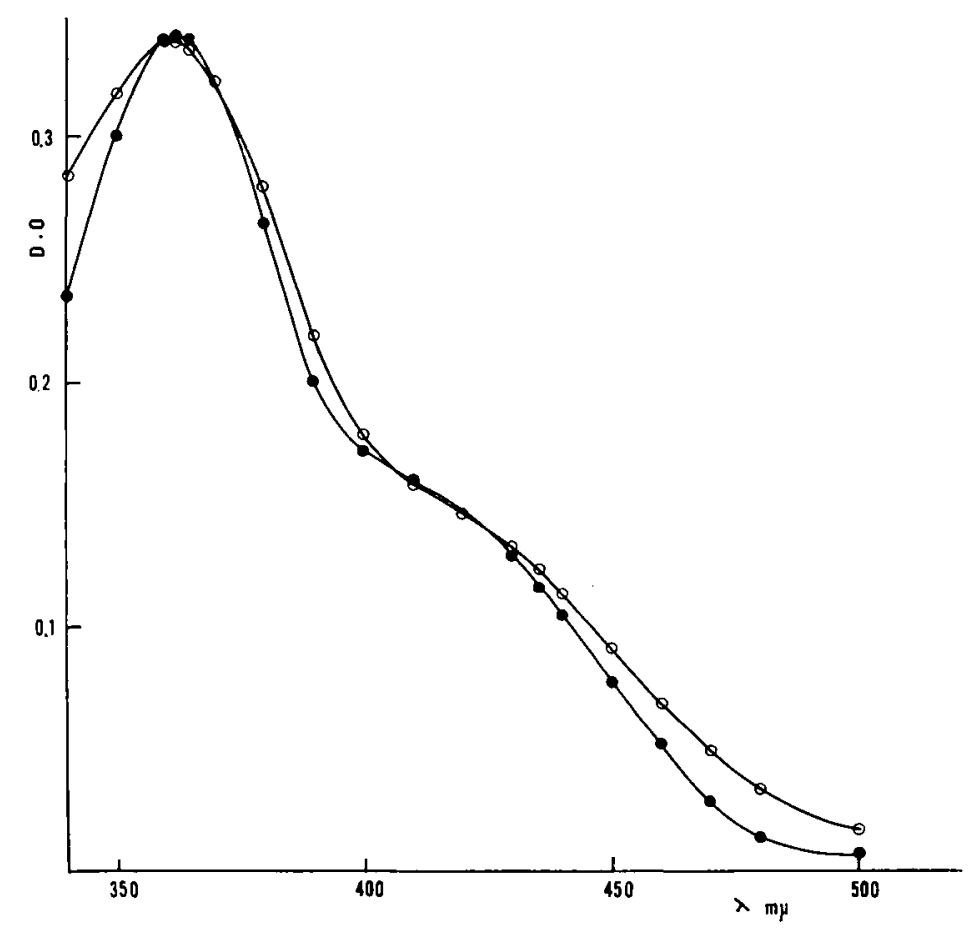

FIG. 4 - Spectres d'absorption de l'sDNP lysine el de la solution obtenue pour le dosage par la méthode II (échantillon $n^{\circ}$ 1)

- . $\mathrm{DNP}$ lysine 0 . Solution 
TABLEAU 3

Résultats obtenus par la méthode III

Lait no 1. Agitation : 2 heures

\begin{tabular}{|c|c|c|c|c|c|c|}
\hline \multirow{4}{*}{$\begin{array}{c}\text { Temps } \\
\text { d'hydroly'se }\end{array}$} & \multicolumn{6}{|c|}{ Lysine " disponible " p. roo. } \\
\hline & \multicolumn{4}{|c|}{ Centrifugation à $4^{\circ} \mathrm{C}$. } & \multirow{2}{*}{\multicolumn{2}{|c|}{ Centrifugation à $25^{\circ} \mathrm{C}$. }} \\
\hline & \multicolumn{2}{|c|}{ Série I } & \multicolumn{2}{|c|}{ Série II } & & \\
\hline & Lect. $362 \mathrm{~m} \mu$ & Lect. $435 \mathrm{~m} \mu$ & Lect. $362 \mathrm{n} \mu$ & Lect. $435 \mathrm{~m} \mu$ & Lect. $362 \mathrm{~m} \mu$ & Lect. $435 \mathrm{~m} \mu$ \\
\hline $15 \mathrm{H}$. & $\begin{array}{l}2,92 \\
3,11\end{array}$ & $\begin{array}{l}2,67 \\
2,87\end{array}$ & & & & \\
\hline 1811. & & & $\begin{array}{l}2,78 \\
2,68\end{array}$ & $\begin{array}{l}2,57 \\
2,50\end{array}$ & $\begin{array}{l}2,58 \\
2,59\end{array}$ & $\begin{array}{l}2,32 \\
2,37\end{array}$ \\
\hline $20 \mathrm{H}$ & $\begin{array}{l}3,04 \\
2,99\end{array}$ & $\begin{array}{l}2,75 \\
2,76\end{array}$ & $\begin{array}{l}2,72 \\
2,80\end{array}$ & $\begin{array}{l}2,50 \\
2,62\end{array}$ & $\begin{array}{l}2,42 \\
2,40\end{array}$ & $\begin{array}{l}2,16 \\
2,16\end{array}$ \\
\hline $24 \mathrm{HI}$. & 3,02 & 2,81 & $\begin{array}{l}2,77 \\
2,92\end{array}$ & $\begin{array}{l}2,58 \\
2,67\end{array}$ & $\begin{array}{l}2,38 \\
2,47\end{array}$ & $\begin{array}{l}2,19 \\
2,28\end{array}$ \\
\hline
\end{tabular}

Les valeurs obtenues après centrifugation à $25^{\circ} \mathrm{C}$ environ laissent supposer qu'il y a des pertes notables au cours de cette opération.

Devant le manque de fidélité des résultats obtenus, nous avons essayé de prolonger le temps d'agitation (tableau IV). Les résultats restent faibles, aussi bien après 3 heures qu'après 2 heures 30 d'agitation.

TABLEAU 4

Résultats obtenus par la méthode III en faisant varier le temps d'agitation

Lait $n^{\circ}$ 1. Centrifugation à $4^{\circ} \mathrm{C}$

\begin{tabular}{|c|c|c|c|c|}
\hline \multirow{2}{*}{$\begin{array}{c}\text { Temps } \\
\text { d'hydrolyse }\end{array}$} & \multicolumn{2}{|c|}{ Agitation $2 \mathrm{~h} 30 \mathrm{mn}}$. & \multicolumn{2}{|c|}{ Agitation $3 \mathrm{~h}$. } \\
\hline & Lecture à $362 \mathrm{~m} \mu$ & Lecture à $435 \mathrm{~m} \mu$ & Lecture à $362 \mathrm{~m} \mu$ & Lecture à $435 \mathrm{~m} \mu$ \\
\hline $16 \mathrm{H}$. & & & $\begin{array}{l}2,63 \\
2,74\end{array}$ & $\begin{array}{l}2,34 \\
2,54\end{array}$ \\
\hline $20 \mathrm{H}$. & & & $\begin{array}{l}2,63 \\
2,53\end{array}$ & $\begin{array}{l}2,37 \\
2,20\end{array}$ \\
\hline $24 \mathrm{H}$. & $\begin{array}{l}2,86 \\
2,78\end{array}$ & $\begin{array}{l}2,80 \\
2,68\end{array}$ & & \\
\hline
\end{tabular}

\section{E. - APPLICATION A DIFFÉRENTS LAITS EN POUDRE}

Io Choix de la méthode.

La quantité d' $\varepsilon$ DNP lysine obtenue par la méthode I est nettement trop faible, et ce composé se trouve en outre mélangé à un autre composé coloré. Cette méthode n'est donc pas applicable telle quelle au dosage de la lysine " disponible » dans les laits en poudre. 
Lorsque les conditions d'hydrolyse sont améliorées la quantité d'ع DNP lysine obtenue reste faible, et variable d'un dosage à l'autre; il y a donc peu de chance pour que les conditions d'hydrolyse soient en cause. L'augmentation du temps de réaction étant inopérante, il semble que la quantité de réactif utilisée (pourtant plus forte que celle préconisée par CARPENTER et EI,IINGER, I955), ou sa concentration, soient trop faibles.

Dans la méthode II, 1'utilisation du méthanol, dans lequel le FDNB est beaucoup plus soluble, permet l'utilisation d'une solution quatre fois plus concentrée, et les résultats obtenus dans ces conditions sont plus élevés et plus reproductibles.

Aussi est-ce la méthode II (avec "centrifugation à $4^{\circ} \mathrm{C}$ et 20 heures d'hydrolyse) qui a été utilisée pour comparer divers échantillons.

\section{$2^{\circ}$ Résultats.}

I,es résultats (tableau V) sont assez voisins de ceux des dosages microbiologiques de la lysine totale, sauf dans le cas du lait no 7 . Les teneurs en lysine "disponible" trouvées pour les échantillons I à 6 sont très élevées, étant donné les pourcentages de perte généralement admis pour le dosage des groupements terminaux au moyen du FDNB (LEVY, I955).

\section{TABLEAU 5}

Résultats obtenus pour différents laits par la méthode II (Lecture à 362 mu) (i)

\begin{tabular}{c|c|c|c}
\hline \hline Echantillon ${ }^{\circ}$ & Lysine " disponible " \% & Lysine " totale " \% & $\begin{array}{c}\text { Lysine " disponible " \% } \\
\text { Lysine totale }\end{array}$ \\
\hline 1 & 3,05 & 3,2 & 95 \\
2 & 3,0 & 3,3 & 91 \\
3 & 2,95 & 3,2 & 92 \\
5 & 2,7 & 2.9 & 93 \\
6 & 2,8 & 2,9 & 96,5 \\
7 & 2,1 & 2,8 & 75 \\
\hline
\end{tabular}

(1) Lait $n^{\circ}$ I : Moyenne des résultats obtenus après des temps d'hydrolyse de $\mathrm{I}_{4}$ à 24 heures. Autres échantillons : temps d'hydrolyse 20 heures.

Si l'on exprime les teneurs en lysine " disponible » et en lysine totale des différents échantillons en $\mathrm{p}$. Ioo des résultats obtenus pour le lait $n^{\circ}$ I (tableau VI), on

$$
\text { TABLEAU } 6
$$

Teneurs en lysine "disponible " et en lysine tolale $p .100$ de la teneur du lait $n^{0} 1$

\begin{tabular}{c|c|c}
\hline \hline Lait $n^{\circ}$ & $\begin{array}{c}\text { Lysine "disponible \#\% } \\
\text { de celle du lait } n^{\circ}\end{array}$ & $\begin{array}{c}\text { Lysine totale \% } \\
\text { de celle du lait } n^{\circ}\end{array}$ \\
\hline 1 & 100 & 100 \\
2 & 98 & 103 \\
3 & 97 & 100 \\
5 & 88,5 & 90,5 \\
6 & 92 & 90,5 \\
7 & 69 & 87,5 \\
\hline
\end{tabular}


constate que 1a détermination de la lysine " disponible " n'apporte pas plus d'information que celle de la lysine totale, sauf dans le cas du lait $n^{\circ} 7$, dont l'altération était perceptible à sa coloration jaunâtre et à sa faible solubilité.

La sensibilité de ces dosages s'avère donc insuffisante pour déceler de faibles altérations. Les échantillons nos 5 et 6 , dont les teneurs en lysine "disponible " et en lysine totale ne sont inférieures que d'environ Io $p$. Ioo à celles du lait no I ( ce qui n'est pas significatif dans le cas des teneurs en lysine "disponible"), se sont révélés beaucoup plus différents de celui-ci dans des expériences en cours sur la libération enzymatique de la 1ysine.

De plus, si la méthode de Schober et PRINZ, modifiée par 1'utilisation d'une hydrolyse chlorhydrique, qui a été appliquée, donne des résultats analytiques cohérents, son application pratique pour des dosages de série est relativement délicate et nécessite l'utilisation d'une verrerie spéciale dont le nettoyage est difficile.

Quoi qu'il en soit, nous ne pourrons conclure sur la validité des résultats obtenus par ces méthodes in vitro qu'après les avoir comparés à des essais in vivo.

Reçu en mai 1960.

\title{
SUMMARY
}

\author{
DETERMINATION OF " AVAILABLE LYSINE" IN DRIED SKIM MILK USING
}

\section{FLUORODINITROBENZENE.}

The application, to dried skim milk, of the determination of "available lysine " by means of dinitrofluorobenzene (FDNB) has been studied. Two techniques were compared, that advocated by CARPENTER and Ellinger (I955) for different animal proteins (method I) and that which SchoBER and PRINZ (I956) have applied to milk (method II), which involves the elimination of the excess FDNB by centrifugation. However, the method of hydrolysis advocated by the latter authors was not used and all the determinations have been carried out after hydrolysis with $6 \mathrm{~N} \mathrm{HCl}$ (CARPENTER and ElLinger). Total lysine was estimated microbiologically using Leuconostoc mesenteroides P 60 (ATCC. 8042).

All the photometric measurements were carried out in I p. Ioo sodium bicarbonate. The standard curves were read at $362 \mathrm{~m} \mu$ (maximum absorption) and at $435 \mathrm{m \mu}$ (the wave length advocated by Carpenter and Ellinger). The determinations were carried out by reference to a standard solution of $\varepsilon$ DNP lysine prepared according to PORTER and SANGER (I948). At 362 m $\mu$ the coloration follows the Beer Law up to $60 \mu$ moles (optical density about I). The minimal concentrations required in the final solution for a good determination are $18 \mu$ moles $/ \mathrm{lat} 362 \mathrm{~m} \mu, 40 \mu$ moles $/ \mathrm{l}$ at $435 \mathrm{~m} \mu$ (fig 2 ).

Methods I and II were compared on the same sample, after various times of hydrolysis (table I and 2). The time of hydrolysis does not significantly influence the results obtained by method II. This method only gives a chromatographically homogenous product (in tert. amyl alcohol-phtalate system). However, the results obtained by method I are low. Moreover, the spectrum of the product obtained by this method is markedly deformed as compared with that of DNP lysine (fig. 3).

In every case there is some difference between the results obtained at the two wave-lengths used.

The utilization of tine reagent of method I under the conditions of method II, tested under various conditions (method III) does not yield quantitative results (table 3 and 4).

As a result of these trials, it was method II which was used for comparing different dried skim milks (table 5). Table 6 shows that this determination does not contribute further information than does that of total lysine, except in the case of the very impaired sample $n^{0} 7$, which was highly coloured and whose solubility was low. The method does not appear to be sufficiently sensitive for the appreciation of slightly deteriorated dried skim milk.

\section{RÉFÉRENCES BIBLIOGRAPHIQUES.}

Bruno D., Carpenter K. J., 1957. A modified procedure for the estimation of " available lysine " in food protein. Biochem. J., 67, I3 p. (Proc).

Carpenter K. J., Ellinger G. M., I955. The estimation of "available lysine "with fluoro-2-4-dinitrobenzène (FDNB). Biochem. J., 61, XI (Proc). 
Carpenter K. J., Ellinger G. M., Munro., M. I. Rolfe E. J., i957. Fish products as protein supplements to cereals. Brit. J. Nutr., 11, I62-173.

Chevillard L., Fauconneau G., Roche J., I952. Dosage microbiologique de la cystine. Application aux produits végétaux utilisés dans l'alimentation du bétail. Ann. Zoot., 3, 3I-44.

DunN M. S., ShankmanN S., BLOCK H., r947. The amino acids requirements of twenty three lactic acid bacteria. J. Biol. Chem., 168, 1-23.

Fauconniau G., I954. Les acides aminés des plantes fourragères. Conférence Européenne des Herbages. Paris, $321-324$.

IIENRY K. M., KON S. K., LEA C. Il., Winte J. C. D., 1948. Deterioration on storage of dried skim milk. J. Dairy Res., 15 292-363.

LfVY A. L., I955, in GLICK, D. Yethods of Biochemical Analysis, vol 2, 360-383. Interscience Publishers, New York.

Mauron J., Motru F., Bujard E., Egli R. H., 1955. The availability of lysine, methionine and tryptophan in condensed milk and milk powder. In vitro digestion studies. Arch. Biochem. Biophys., 59, 433-45I.

Porter R. R., SANGER H., I948. The free amino groups of haemoglobins. Biochem. I., 42, $287-94$.

Sciober R., Prinz I., I956. Bestimmung des Lysingehalts in Milchproben nach der DNP-Methode, in Abhängigkeit von ihrer Erhitzung. Milchwissenschaft, 11, 466-68. 